\title{
Potenciais interações entre medicamentos alopáticos e fitoterápicos/ plantas medicinais no Município de Rondonópolis - MT
}

\author{
Potential interactions between allopathic and phytotherapy mediciation/medicinal \\ plants in the Municipality of Rondonópolis
}

\begin{abstract}
Raquel Aparecida Rodrigues Nicácio ${ }^{1}$, Graziele Ferreira Pinto ${ }^{1}$, Fernanda Rocha Anjos de Oliveira ${ }^{1}$, Débora Aparecida da Silva Santos ${ }^{2}$, Magda de Mattos ${ }^{3}$, Letícia Silveira Goulart ${ }^{4 *}$

${ }^{1}$ Enfermeira pela Universidade Federal de Rondonópolis (UFR); ${ }^{2}$ Doutora em Recursos Naturais, Professora do Curso de Enfermagem da UFR; ${ }^{3}$ Doutora em Educação, Professora do Curso de Enfermagem da UFR; ${ }^{4}$ Doutora em Ciências, Professora do Curso de Enfermagem da UFR, Rondonópolis, MT
\end{abstract}

\begin{abstract}
Resumo
Introdução: no Brasil, a utilização de fitoterápicos e plantas medicinais é uma prática amplamente difundida, todavia, o consumo destes compostos em associação com medicamentos alopáticos caracteriza um risco à saúde devido às potenciais interações medicamentosas e seus efeitos. Objetivo: analisar as potenciais interações envolvendo fitoterápicos e plantas medicinais com medicamentos alopáticos na população de Rondonópolis, MT. Metodologia: trata-se de um estudo transversal de base populacional com 370 participantes. Os dados foram coletados em visitas domiciliares com um instrumento estruturado e padronizado. Para identificar as potenciais interações foi utilizada a base de dados Medscape ${ }^{\circ}$ e a literatura nacional e internacional. Resultados: 131 (35,40\%) indivíduos informaram consumir plantas medicinais e ou fitoterápicos concomitante a medicamentos alopáticos. A interação entre fitoterápicos e medicamentos alopáticos mais frequente foi entre Passiflora incarnata e cinarizina, para plantas medicinais foi entre hortelã e sinvastatina. As consequências mais prevalentes decorrentes das interações foram a intensificação da depressão do Sistema Nervoso Central, o aumento da anticoagulação e o risco de hipoglicemia. Conclusão: os dados analisados no presente estudo possibilitaram identificar potenciais interações existentes entre medicamentos alopáticos e plantas medicinais/fitoterápicos na população de Rondonópolis-MT e apontam para a necessidade de se estimular o uso racional da fitoterapia no âmbito da saúde pública. Palavras-chave: Interações de medicamentos. Plantas medicinais. Medicamentos fitoterápicos.
\end{abstract}

\begin{abstract}
Introduction: in Brazil, the use of phytotherapy medication and medicinal plants is a widespread practice. However, the consumption of these compounds in association with allopathic medicinal products is a health risk due to potential drug interactions and their effects. Objective: to analyze the potential interactions involving phythotherapy medication and medicinal plants with allopathic drugs in the population of Rondonópolis, state of MT. Methodology: this is a cross-sectional population-based study with 370 participants. Data were collected in home visits with a structured and standardized instrument. Medscape database and national and international literature were used to identify potential interactions. Results: 131 (35, 40\%) individuals reported consuming medicinal plants and/or phytotherapy medication concomitantly with allopathic drugs. The most frequent interaction between phytotherapeutic and allopathic drugs was between Passiflora incarnata and cinnarizine, and in medicinal plants, it was between peppermint and simvastatin. The most prevalent consequences of the interactions were intensification of central nervous system depression, increased anticoagulation and risk of hypoglycemia. Conclusion: data analyzed in the present study enabled the identification of potential interactions between allopathic medicines and herbal plants/phytotherapeutic medication in the population of Rondonópolis (MT), and demonstrated the need to stimulate the rational use of phytotherapy in public health.
\end{abstract}

Keywords: Drug interactions. Plants, medicinal. Phytotherapeutic drugs.

\section{INTRODUÇÃO}

As plantas medicinais e medicamentos fitoterápicos são reconhecidos no Brasil como uma alternativa terapêutica para grande parte da população, sendo comumente utilizados de forma empírica e tradicional ${ }^{1}$. Cerca de $82 \%$ da população brasileira utiliza produtos à base de plantas medicinais para cuidados com a saúde,

Correspondente/Corresponding: *Letícia Silveira Goulart - End: Avenida dos Estudantes, 5055 - Cidade Universitária - Rondonópolis - MT, CEP: 78736-900 - Tel: - E-mail: Igoulart77@yahoo.com.br seja pelos conhecimentos e tradições populares acumulados ou pela percepção de que produtos naturais são inofensivos e desprovidos de efeitos adversos ${ }^{2}$. Todavia, são crescentes as preocupações acerca da segurança em sua utilização e ao risco de interações medicamentosas.

A fitoterapia é caracterizada pelo uso de plantas medicinais em diferentes formas farmacêuticas ${ }^{3}$. Os medicamentos fitoterápicos são obtidos através do emprego de matérias-primas vegetais com efeito farmacológico, conhecimento da eficácia e riscos de seu uso, assim como pela reprodutibilidade e constância 
de sua qualidade, enquanto as plantas medicinais são utilizadas há milênios como forma de tratamento de doenças, na forma in natura, na maioria das vezes, por automedicação, sem prescrição, orientação e/ou o acompanhamento, sendo esta, de fácil acesso e custo mais acessível a grande parcela da população ${ }^{4}$.

Os medicamentos alopáticos são considerados um bem essencial à saúde e uma importante ferramenta terapêutica, responsável por parte da melhoria da qualidade e expectativa de vida da população $0^{5}$. Eles contêm substâncias ativas com propriedades terapêuticas reconhecidas cientificamente, que fazem parte da composição do produto, denominadas fármacos, drogas ou princípios ativos ${ }^{6}$. Para muitos pacientes, o uso de um único medicamento não é suficiente, e quando dois ou mais são prescritos podem interagir negativamente ${ }^{7}$.

As interações medicamentosas ocorrem quando o efeito de um medicamento é alterado pelo uso concorrente de alguma substância, aumentando ou reduzindo o efeito terapêutico ou tóxico de um ou de outro ${ }^{3}$. Os usuários de plantas medicinais e fitoterápicos são predominantemente adultos e idosos que associam seu uso a medicamentos alopáticos como forma de tratamento de doenças crônicas, aumentando as chances de complicações a saúde, podendo resultar até mesmo em morte, hospitalização, injúria permanente ou insucesso terapêutico ${ }^{1,7}$.

O risco da ocorrência de interações envolvendo plantas medicinais ou fitoterápicos pode ser maior que o de interações entre os medicamentos alopáticos, pois esses normalmente contêm substâncias químicas únicas, enquanto quase todas as plantas contêm misturas de substâncias complexas e farmacologicamente ativas, podendo causar alterações relevantes nas concentrações plasmáticas dos medicamentos, e consequentemente trazer riscos á saúde ${ }^{7}$.

Essas interações podem ser classificadas em farmacocinéticas e farmacodinâmicas. Segundo Salvi e Heuser, no primeiro caso a interação é representada principalmente por condições em que o fitoterápico modifica a absorção ou biotransformação do fármaco, enquanto que na interação farmacodinâmica se refere nas condições em que ocorre sinergia ou antagonismo em relação a um efeito particular, podendo ocorrer com o fitoterápico em sua forma original ou até metabólitos formados pelo organismo ${ }^{8}$.

Desta forma, apesar da sucessiva procura pelas práticas integrativas medicamentosas e reconhecimento da eficácia desta terapêutica, os estudos acerca da fitoterapia ainda são deficientes no Brasil, sendo necessário o desenvolvimento de pesquisas nesta temática, tornando as práticas fitoterápicas mais seguras e eficazes ${ }^{9}$. Neste contexto, o objetivo deste estudo foi identificar os potenciais riscos de interações medicamentosas entre e fitoterápicos e ou plantas medicinais com medicamentos alopáticos em um município do sudeste de Mato Grosso.

\section{METODOLOGIA}

Trata-se de um estudo transversal de base populacional. A amostra foi constituída por indivíduos com idade igual ou superior a 20 anos, residentes em Rondonópolis, MT. O processo de amostragem ocorreu por conglomerados em dois estágios, sendo a unidade primária de amostragem o setor censitário e a secundária, o domicílio. No primeiro estágio, foram sorteados 37 setores censitários com probabilidade proporcional ao tamanho - expresso pelo número de domicílios existentes em cada um deles segundo a Fundação Instituto Brasileiro de Geografia e Estatística ${ }^{10}$. No segundo estágio, uma amostra sistemática de domicílios foi sorteada em cada setor censitário, com base na listagem dos domicílios previamente arrolados. Nos domicílios com mais de um adulto presente no momento da coleta de dados foi realizado um sorteio com o uso de uma tabela de números aleatórios.

Os dados foram coletados através de visitas domiciliárias, no período de janeiro a março de 2018, utilizando um instrumento do tipo formulário semi-estruturado, previamente testado. Os participantes do estudo foram questionados acerca de todos os medicamentos e plantas medicinais utilizados nos últimos 15 dias. Para a validação do uso de medicamentos alopáticos e fitoterápicos, foi utilizada a metodologia padrão instituída por Landry et al. ${ }^{11}$, tal técnica consiste em solicitar aos participantes do estudo a embalagem, receita, bula ou blister de produtos farmacêuticos utilizados, visando evitar omissão, em geral por esquecimento de medicamentos em uso, e, além disso, para assegurar a veracidade das especialidades farmacêuticas informadas.

Para identificar as interações decorrentes do uso de plantas medicinais e fitoterápicos concomitante a medicamentos alopáticos foram utilizadas a base de dados Medscape ${ }^{\oplus 12}$ e relatos na literatura ${ }^{4,12-14}$.

As variáveis de estudo foram idade, gênero, cor auto-referida, escolaridade, renda, inserção no mercado de trabalho, situação conjugal, utilização de plantas medicinais, fitoterápicos e de medicamentos alopáticos nos últimos 15 dias anteriores à entrevista.

Os usuários que concordaram em participar da pesquisa receberam, para assinatura prévia, o Termo de Consentimento Livre e Esclarecido em duas vias, sendo que uma foi retida com o pesquisador e outra entregue ao participante. Esta pesquisa faz parte do projeto intitulado "Práticas Integrativas e Complementares por Profissionais e Usuários do Sistema Único de Saúde em Município do estado de Mato Grosso" e foi aprovado pelo Comitê de Ética em Pesquisa da UFMT/Rondonópolis n. 2.354.295. Desta maneira, foram respeitados todos os aspectos éticos de pesquisa com seres humanos, de acordo com a Resolução n. 466/2012. ${ }^{15}$ 


\section{RESULTADOS}

Participaram do estudo 370 indivíduos, sendo que $131(35,40 \%)$ informaram consumir plantas medicinais e ou fitoterápicos concomitante a medicamentos alopáticos, estes, em sua maioria ( $n=76,58,02 \%)$ tinham idade igual ou superior a sessenta anos, pertenciam ao gênero feminino ( $n=106,80,92 \%)$, se auto declararam de cor parda ( $n=60,45,81 \%)$, eram casados ( $n=79,60,30 \%)$, economicamente ativos ( $n=85,64,89 \%)$ tinham renda de até dois salários mínimos $(n=88,67,17 \%)$ e relataram ter estudado até 8 anos ( $n=75,57,26 \%)$.

A prevalência de consumo de fitoterápicos foi de $7,30 \%(n=27)$ sendo que a utilização destes medicamentos concorrente à alopáticos foi relatada por $21(5,67 \%)$ dos participantes da pesquisa. Os medicamentos fitoterápico mais consumidos foram Ginkgo biloba (21,74\%), Camellia sinensis (8,69\%), Harpagophytum procumbens $(8,69 \%)$, Passiflora incarnata $(8,69 \%)$ e Valeriana officinalis (8,69\%). A tabela 1 descreve os fitoterápicos consumidos pela população estudada.

Tabela 1 - Fitoterápicos utilizados pela população de Rondonópolis - MT, 2018.

\begin{tabular}{lcc}
\hline \multicolumn{1}{c}{ Fitoterápicos } & $\begin{array}{c}\text { Número de } \\
\text { citações }\end{array}$ & $\%$ \\
\hline Ginkgo biloba & 5 & $21,74 \%$ \\
Camellia sinensis & 2 & $8,69 \%$ \\
Harpagophytum procumbens & 2 & $8,69 \%$ \\
Passiflora incarnata & 2 & $8,69 \%$ \\
Valeriana officinalis & 2 & $8,69 \%$ \\
Equisetum arvense & 1 & $4,35 \%$ \\
Curcuma longa & 1 & $4,35 \%$ \\
Passiflora incarnata+ Hypericum perfo- & & \\
ratum & 1 & $4,35 \%$ \\
Passiflora incarnata+lgnetia amara+Coffe & & \\
cruda & 1 & $4,35 \%$ \\
Cynara scolymus & 1 & $4,35 \%$ \\
Pneumus boldus molina & 1 & $4,35 \%$ \\
Actea remosa & 1 & $4,35 \%$ \\
Trifolium Pratense & 1 & $4,35 \%$ \\
Panax Ginseng & 1 & $4,35 \%$ \\
Isoflavona & 23 & $4,35 \%$ \\
Total & $100 \%$ \\
\hline Eonte: Autores & & \\
\hline & 1 & \\
\hline
\end{tabular}

Fonte: Autores

No que se refere ao uso de plantas medicinais, estas foram declaradas por 157 (42,43\%) participantes da pesquisa e $125(33,78 \%)$ as utilizaram concomitantemente a medicamentos. As plantas medicinais mais citadas foram erva cidreira $(23,08 \%)$, hortelã $(14,98 \%)$, e boldo $(10,53 \%)$ (tabela 2).
Tabela 2 - Principais plantas medicinais utilizadas pela população de Rondonópolis, MT. 2018.

\begin{tabular}{lcc}
\hline Plantas Medicinais & N & \% \\
\hline Erva-cidreira & 57 & $23,08 \%$ \\
Hortelã & 37 & $14,98 \%$ \\
Boldo & 26 & $10,53 \%$ \\
Camomila & 22 & $8,91 \%$ \\
Alecrim & 18 & $7,29 \%$ \\
Canela & 17 & $6,88 \%$ \\
Folha de limão & 11 & $4,45 \%$ \\
Gengibre & 10 & $4,05 \%$ \\
Capim Santo & 8 & $3,24 \%$ \\
Canela de velho & 7 & $2,83 \%$ \\
Outros & 34 & $13,76 \%$ \\
Total & 247 & $100 \%$ \\
\hline
\end{tabular}

Fonte: Autores

Os medicamentos mais consumidos em associação com fitoterápicos e ou plantas medicinais foram os que atuam no sistema cardiovascular $(41,85 \%)$, sistema nervoso $(15,98 \%)$ e aparelho digestório $(13,42 \%)$, sendo que Losartana (10,54\%), Hidroclorotiazida (6,38\%) e Metformina $(5,43 \%)$ foram os mais utilizados.

As interações entre fitoterápicos e medicamentos alopáticos encontradas na população em estudo foram entre Passiflora incarnata e cinarizina ( $n=2)$ Ginkgo biloba e atenolol $(n=1)$ e Valeriana officinalis e amitriptilina $(n=1)$. Estes dados estão representados na Tabela 3.

Tabela 3 - Potenciais interações entre fitoterápicos e medicamentos alopáticos identificados na população de Rondonópolis - MT. 2018

\begin{tabular}{|c|c|c|c|}
\hline Fitoterápico & $\begin{array}{c}\text { Fármaco que } \\
\text { pode ocorrer } \\
\text { interação }\end{array}$ & $\begin{array}{c}\text { Potenciais } \\
\text { consequências }\end{array}$ & $\begin{array}{c}\text { Número de } \\
\text { potenciais } \\
\text { interações }\end{array}$ \\
\hline $\begin{array}{l}\text { Passiflora } \\
\text { incarnata }\end{array}$ & Cinarizina $^{4,12,13}$ & $\begin{array}{l}\text { Aumenta De- } \\
\text { pressão do SNC }\end{array}$ & $2(50 \%)$ \\
\hline Ginkgo biloba & Atenolol ${ }^{2,13}$ & $\begin{array}{l}\text { Bradicardia e } \\
\text { hipotensão }\end{array}$ & $1(25 \%)$ \\
\hline $\begin{array}{l}\text { Valeriana } \\
\text { officinalis }\end{array}$ & $\begin{array}{l}\text { Amitriptili- } \\
\text { na }^{12,13}\end{array}$ & $\begin{array}{l}\text { Aumenta De- } \\
\text { pressão do SNC }\end{array}$ & $1(25 \%)$ \\
\hline Total & & & $4(100 \%)$ \\
\hline
\end{tabular}

Fonte: Autores

As interações mais frequentemente observadas entre plantas medicinais e medicamentos alopáticos foram entre hortelã e sinvastatina $(n=5)$, boldo e hidroclorotiazida $(n=3)$ e entre erva cidreira e fluoxetina $(n=3)$. A tabela 4 apresenta estes resultados. 
Tabela 4 - Potenciais interações entre plantas medicinais e medicamentos alopáticos identificadas na população de Rondonópolis-MT, 2018.

\begin{tabular}{|c|c|c|c|}
\hline Planta Medicinal & Fármaco que pode ocorrer interação & Potenciais consequências & n (\%) \\
\hline \multirow[t]{4}{*}{ Gengibre } & Diclofenaco $^{12}$ & Aumenta anticoagulação & $2(5,41 \%)$ \\
\hline & Ácido Acetilsalicílico ${ }^{12}$ & Aumenta anticoagulação & $1(2,70 \%)$ \\
\hline & Metformina ${ }^{12}$ & Potencial hipoglicemia & $1(2,70 \%)$ \\
\hline & Losartana $^{12}$ & Potencial hipotensão & $1(2,70 \%)$ \\
\hline \multirow[t]{4}{*}{ Canela } & Glibenclamida $^{12}$ & Potencial hipoglicemia & $1(2,70 \%)$ \\
\hline & Ácido Acetilsalicílico ${ }^{12}$ & Aumenta anticoagulação & $1(2,70 \%)$ \\
\hline & Metformina $^{12}$ & Potencial hipoglicemia & $1(2,70 \%)$ \\
\hline & Diclofenaco ${ }^{12}$ & Aumenta anticoagulação & $1(2,70 \%)$ \\
\hline \multirow[t]{2}{*}{ Chá verde } & Dipirona sódica+mucato de isomepteno+cafeína ${ }^{12,13}$ & Aumento do estímulo do SNC & $1(2,70 \%)$ \\
\hline & Etinilestradiol $^{12,13}$ & Aumenta tempo de meia vida do Chá verde & $1(2,70 \%)$ \\
\hline \multirow[t]{3}{*}{ Erva doce } & Ibuprofeno ${ }^{12}$ & Aumenta anticoagulação & $1(2,70 \%)$ \\
\hline & Naproxeno ${ }^{12}$ & Aumenta anticoagulação & $1(2,70 \%)$ \\
\hline & Amitriptilina $^{12}$ & Aumento da depressão do SNC & $1(2,70 \%)$ \\
\hline \multirow[t]{2}{*}{ Boldo } & Hidroclorotiazida ${ }^{4,13}$ & Aumenta efeito da Hidroclorotiazida & $3(8,12 \%)$ \\
\hline & Ácido Acetilsalicílico ${ }^{4,13}$ & Aumenta anticoagulação & $1(2,70 \%)$ \\
\hline \multirow[t]{4}{*}{ Erva Cidreira } & Fluoxetina ${ }^{13,14}$ & Intensificação da ação depressora do SNC & $3(8,12 \%)$ \\
\hline & Clonazepam ${ }^{13,14}$ & Intensificação da ação depressora do SNC & $2(5,41 \%)$ \\
\hline & Amitriptilina ${ }^{13,14}$ & Intensificação da ação depressora do SNC & $1(2,70 \%)$ \\
\hline & Bromazepam ${ }^{13,14}$ & Intensificação da ação depressora do SNC & $1(2,70 \%)$ \\
\hline \multirow[t]{2}{*}{ Hortelã } & Sinvastatina ${ }^{4,13}$ & Aumenta efeito da sinvastatina & $5(13,53 \%)$ \\
\hline & Omeprazol ${ }^{4,13}$ & Diminui efeito do omeprazol & $1(2,70 \%)$ \\
\hline \multirow[t]{2}{*}{ Babosa } & Glibenclamida $^{13,14}$ & Potencial hipoglicemia & $1(2,70 \%)$ \\
\hline & Furosemida ${ }^{13,14}$ & Risco de hipocalemia & $1(2,70 \%)$ \\
\hline \multirow[t]{3}{*}{ Camomila } & Fluoxetina $^{13}$ & Intensificação da ação depressora do SNC & $2(5,41 \%)$ \\
\hline & Clonazepam ${ }^{13}$ & Intensificação da ação depressora do SNC & $1(2,70 \%)$ \\
\hline & Amitriptilina $^{13}$ & Intensificação da ação depressora do SNC & $1(2,70 \%)$ \\
\hline Total & & & 37 (100\%) \\
\hline
\end{tabular}

Fonte: Autores

\section{DISCUSSÃO}

A Política Nacional de Plantas Medicinais e Fitoterápicos representou um incentivo à utilização de fitoterápicos no Brasil, uma vez que, propõe ampliar as opções terapêuticas aos usuários, com garantia de acesso a plantas medicinais, fitoterápicos e serviços relacionados à fitoterapia, com segurança, eficácia e qualidade, na perspectiva da integralidade da atenção à saúde ${ }^{6}$. Neste inquérito, $7,30 \%$ da população informou consumir fitoterápicos nos 15 dias que antecederam a pesquisa, um valor próximo ao encontrado em uma pesquisa brasileira ${ }^{16}$ realizada com idosos (10,6\%). A baixa prevalência de consumo de fitoterápicos observada pode ser justificada pelo fato de que neste inquérito foram incluídos apenas os medicamentos consumidos nos últimos quinze dias, todavia, a utilização deste período recordatório, garante a confiabilidade dos dados coletados.

Os medicamentos fitoterápicos são consumidos por automedicação ou por prescrição médica e o perfil tóxi- co da maioria destes compostos é desconhecido, o que pode acarretar no surgimento de efeitos indesejáveis ${ }^{17}$. Conhecer as possíveis interações medicamentosas com fitoterápicos poderá contribuir com a redução dos riscos à saúde das populações. No município de Rondonópolis, MT a prevalência de uso de fitoterápicos associados a medicamentos alopáticos foi de 5,67\%. Em Ijuí, RS, $15,25 \%$ das mulheres no climatério estudadas utilizavam este tipo de associação ${ }^{7}$. Em lapu, MG, 17\% dos idosos do município consumiam fitoterápicos juntamente com medicamentos alopáticos ${ }^{18}$. Cabe ressaltar, que estudos que avaliam o consumo e a prevalência de associação entre medicamentos fitoterápicos e alopáticos são escassos, reforçando a necessidade de ampliação deste tipo de pesquisas, sobretudo na Estratégia de Saúde da Família, a fim de se fortalecer a Política Nacional de Plantas Medicinais e Fitoterápicos.

A utilização segura de fitoterápicos e plantas medicinais está vinculada a prática de orientação adequada 
acerca dos riscos relativos ao consumo concomitantemente a medicamentos alopáticos. A falta de informação, a polifarmácia, automedicação e a não comunicação ao profissional de saúde sobre utilização destes produtos nas consultas são fatores de risco para interações farmacológicas ${ }^{19}$. As interações entre fitoterápicos e medicamentos alopáticos observadas na população de Rondonópolis foram entre Passiflora incarnata e Cinarizina, Ginkgo biloba e atenolol e Valeriana officinalis e amitriptilina. Marliére e colaboradores identificaram que as principais interações com fitoterápicos envolviam Ginkgo biloba na composição ${ }^{16}$. As interações entre fitoterápicos e medicamentos podem causar eventos adversos com graves consequências à saúde, sendo considerado um sério problema de saúde pública, porém estas ainda são pouco divulgadas a população, necessitando desta forma a promoção de conhecimento aos profissionais de saúde para que os mesmos saibam prescrever e praticar a fitoterapia de forma congruente e segura ${ }^{20}$.

Um total de 157 (42,43\%) pesquisados relatou consumir plantas medicinais, pesquisas brasileiras apontam para valores que variam de $21 \%$ a $92 \%{ }^{18,19,21-25}$. A divergência dos resultados é atribuída ao fato de que muitas pesquisas englobam diferentes grupos populacionais. Apesar do amplo conhecimento sobre plantas medicinais, ainda se faz necessário a ampliação de pesquisas sobre este tema, principalmente, levando-se em consideração os riscos à saúde que uso incorreto destes produtos pode ocasionar. Somado a isto, a realização de pesquisas com plantas medicinais, poderá contribuir para a obtenção de novos medicamentos fitoterápicos e fitofármacos, ampliando assim, o seu acesso à população ${ }^{26}$.

Uma frequência de $33,78 \%$ dos entrevistados informou consumir medicamentos alopáticos em associação com plantas medicinais. Dados da literatura descrevem frequências de $55,9 \%$ na região centro-norte do Estado do Rio de janeiro ${ }^{19}$ e de $86,2 \%$ em Uberlândia, Minas Gerais ${ }^{24}$. Na presente pesquisa foram identificadas 37 (28,24\%) interações entre plantas medicinais e medicamentos alopáticos, com predomínio dos fármacos com ação hipolipemiante, diurética e ansiolítica/antidepressiva. Em estudo similar realizado em Minnesota, EUA, pesquisadores identificaram 107 (29\%) interações com importância clínica, sendo que os medicamentos que tiveram maior número de interações foram os antitrombóticos, antidepressivos, sedativos e antidiabéticos. ${ }^{27} \mathrm{~A}$ interação mais frequente ocorreu entre hortelã e sinvastatina. Estudos mostram que a hortelã (Mentha piperita) pode inibir a citocromo P450 3A4, levando a um potencial aumento dos níveis sanguíneos de fármacos que são metabolizados pelo mesmo, como a sinvastatina, aumentando, desta forma, o risco de miopatias. ${ }^{4,28}$ Neste sentido, cabe aos profissionais prescritores incorporar a prática de se avaliar toda a medicação consumida, assim como as plantas medicinais, como parte de sua anamnese, a fim de se reduzir os riscos associados a interações medicamentosas.

\section{CONCLUSÃO}

Os dados analisados no presente estudo possibilitaram identificar potenciais interações existentes entre medicamentos alopáticos e plantas medicinais/fitoterápicos na população de Rondonópolis-MT. Observou-se que a população em questão teve um alto índice de casos potenciais de interações entre plantas medicinais e medicamentos alopáticos. Deste modo, ressalta-se a importância dos profissionais de saúde conhecerem e conscientizarem a população sobre o risco deste tipo de interação medicamentosa, contribuindo assim para a promoção do uso racional de medicamentos.

\section{REFERÊNCIAS}

1. ALEXANDRE, R. F; BAGATINI, F.; SIMÕES, C. M. O. Interações entre fármacos e medicamentos fitoterápicos à base de ginkgo ou ginseng. Rev. Bras. Farmacogn., São Paulo, v. 18, n. 1, p. 117-126, 2008.

2. RODRIGUES, A. G.; SIMONI, C. Plantas medicinais no contexto de políticas públicas. Informe Agropecuário. Belo Horizonte, v. 31, n. 255, p. 7-12, 2010.

3. DIAS, E. C. M et al. Uso de fitoterápicos e potenciais riscos de interações medicamentosas: Reflexões para prática segura. Rev. Baiana de Saúde Pública., Salvador, v. 41, n. 2, p. 297-307, 2017.

4. NICOLETTI, M. A. et al. Principais Interações no uso de medicamentos fitoterápicos. Infarma, Brasília, v. 19, n. 1, p. 32-40, 2007.

5. ARRAIS, P. S. D. et al. Prevalência e fatores determinantes do consumo de medicamentos no Município de Fortaleza, Ceará, Brasil. Cad. Saúde Pública, Rio de Janeiro, v. 21, n. 6, p. 1737-1746, 2005.

6. BRASIL. Ministério da Saúde. Secretaria de Atenção à Saúde. Departamento de Atenção Básica. Práticas integrativas e complementares: plantas medicinais e fitoterapia na Atenção Básica. Brasília: Ministério da Saúde, 2012. (Cadernos de Atenção básica, n. 31)

7. GELATTI, G. T.; OLIVEIRA, K. R.; COLET, C. F. Potenciais interações relacionadas ao uso de medicamentos, plantas medicinais e fitoterápicos em mulheres no período do climatério. J. Research: Fundamental Care Online, Rio de Janeiro, v. 8, n. 2, 2016.

8. SALVI, R. S.; HEUSER, E. D. Interações Medicamentos x Fitoterápicos: em busca de uma prescrição racional. EDIPUC - RS, Rio Grande do Sul, p. 116, 2008.

9. SANTOS, R. L. et al. Análise sobre a fitoterapia como prática integrativa no Sistema Único de Saúde. Rev. Bras. PI. Med., Botucatu, v. 13, n. 4, p. 486-491, 2011.

10. IBGE. Dados sobre População do Brasil, PNAD (Pesquisa Nacional por Amostra de Domicílios). 2001.

11. LANDRY, J. A. et al. Validation of two methods of data collection of self-reported medicine among the elderly. Gerontologist, Washington, v. 28 , n. 5 , p. $672-676,1988$.

12. MEDSCAPE. Drug interactions checker. Disponível em: http://www. medscape.com/pharmacists. Acesso em: 10 Oct. 2018.

13. POSADZKI, P.; WATSON, L. K.; ERNST, E. Adverse effects of herbal medicines: an overview of systematic reviews. Clin Med, London, v. 13, n. 1, p. 7-12, 2013

14. NICOLETTI, M. A. et al. Fitoterápicos - principais interações medicamentosas. São Paulo: Associação Nacional de Farmacêuticos Magistrais, 2012. p. 118. 
15. BRASIL. Conselho Nacional de Saúde. Resolução no 466 de 12 dez. 2012. Trata de pesquisas em seres humanos e atualiza a resolução 196. Diário Oficial [da] União. 12 dez. 2012.

16. MARLIÉRE, L. D. P. et al. Utilização de fitoterápicos por idosos: resultados de um inquérito domiciliar em Belo Horizonte (MG), Brasil. Rev. Bras. Farmacogn., São Paulo, v. 18, p. 754-760, 2008.

17. SILVEIRA, P. F.; BANDEIRA, M. A. M.; ARRAIS, P. S. D. Farmacovigilância e reações adversas às plantas medicinais e fitoterápicos: uma realidade. Rev. Bras. Farmacogn., São Paulo, v. 18, n. 4, p. 618-626, 2008.

18. SILVA, N. C. S. Tudo que é natural não faz mal? Investigação sobre o uso de plantas medicinais e medicamentos fitoterápicos por idosos, na cidade de lapu - leste de Minas Gerais. Única Cadernos Acadêmicos, Ipatinga, v. 2, n. 2, p. 1-12, 2016.

19. VEIGA JÚNIOR, V. F. Estudo do consumo de plantas medicinais na Região Centro-Norte do Estado do Rio de Janeiro: aceitação pelos profissionais de saúde e modo de uso pela população. Rev. Bras. Farmacogn., São Paulo, v. 18, n. 2, p. 308-313, 2008.

20. BALBINO, E. E.; DIAS, M. F. Farmacovigilância: um passo em direção ao uso racional de plantas medicinais e fitoterápicos. Rev. Bras. Farmacogn., São Paulo, v. 20, n. 6, p. 992-1000, 2010.

21. FERREIRA, R. S. et al. Utilização de fitoterápicos pela população atendida no "Programa Saúde da Família", Realengo, RJ. R. Pesq.: Cuid. Fundam., Rio de Janeiro, v. 2, p. 40-43, 2010.
22. ZENI, A. L. B. et al. Utilização de plantas medicinais como remédio caseiro na Atenção Primária em Blumenau, Santa Catarina, Brasil. Ciên. Saúde Colet., Rio de Janeiro, v. 22, n. 8, p. 2703-2712, 2017.

23. BRASILEIRO, B. G. et al. Plantas medicinais utilizadas pela população atendida no "Programa de Saúde da Família", Governador Valadares, MG, Brasil. Rev. Bras. Ciên. Farm., São Paulo, v. 44, n. 4, p. 629-636, 2008.

24. MACHADO, H. L. et al . Pesquisa e atividades de extensão em fitoterapia desenvolvidas pela Rede FitoCerrado: uso racional de plantas medicinais e fitoterápicos por idosos em Uberlândia-MG. Rev. Bras. Plantas Med., Botucatu, v. 16, n. 3, p. 527-533, 2014.

25. SCHWAMBACH, K. H.; AMADOR, T. A. Estudo da Utilização de Plantas Medicinais e Medicamentos em um Município do Sul do Brasil. Lat. Am. J. Pharm., Buenos Aires, v. 26, n. 4, p. 602-608, 2007.

26. CORDEIRO, J. M. P.; FELIX, L. P. Conhecimento botânico medicinal sobre espécies vegetais nativas da caatinga e plantas espontâneas no agreste da Paraíba, Brasil. Rev. Bras. Plantas Med., Botucatu, v. 16, n. 3, p. 685-692, 2014.

27. SOOD, A. et al. Potential for Interactions Between Dietary Supplements and Prescription Medications. Am. J. Med., Arizona, v. 121, n. 3, p. 207-211, 2008.

28. BRASIL. Ministério da Saúde. Agência Nacional de Vigilância Sanitária. Monografia da espécie Mentha x piperita L. (Hortelã pimenta). Brasília: MS, 2015.

Submetido em: $23 / 08 / 2019$

Aceito em: 08/09/2020 